\title{
A Gurson Model Improved by Cohesive Traction-Separation Law to Realize Transition from Ductile to Brittle Fracture*
}

\author{
by KAGIMURA Takuya **, SHINTAKU Yuichi***, TSUTSUMI Seiichiro**** and TERADA Kenjiro*****
}

\begin{abstract}
The objective of this study is to improve Gurson model by combining with cohesive traction-separation law to realize crack propagation associated with transition from ductile to brittle fracture. To embed the cohesive cracks into the Gurson model, five kinds of conditional equations are solved for the crack opening displacement and the plastic strain. One of the conditional equations correspond to the local balance equations between the cohesive tractions and the principal stresses and the others are the yield function, the isotropic hardening law, evolutional equation of void volume fraction and inequality constraint. The enhanced Gurson model allows us to represent the nucleation and propagation of the ductile crack along with the void nucleation and growth. Moreover, it is realized by the embedded cohesive traction-separation law that the stress rapidly drops down when the crack accelerates due to the transition from the ductile to brittle fracture. Throughout the numerical examples at several temperatures, it is confirmed that the proposed model enables us to realize load-displacement curves depending on temperature along with the ductile-brittle transition. Also, the proposed model has represented changes of crack propagation rate and void volume fraction by depending on temperature. Furthermore, the proposed model has capability of reproducing the crack propagation associated with the transition from the ductile to brittle fracture at $-60{ }^{\circ} \mathrm{C}$.
\end{abstract}

Key Words: Ductile to Brittle Transition, Gurson Model, Cohesive Traction-Separation Law, Nucleation and Propagation of Crack, CohesiveTraction Embedded Damage Model

\section{Introduction}

It is well known that metallic materials suffer ductile brittle transition as lower temperature. Also, after the crack initially nucleates and propagates by the ductile fracture, it sometimes transitions to the brittle fracture. It is caused by the degradation of the fracture toughness due to nucleation and growth of voids under the plastic deformation. To prevent the rapid propagation of the brittle crack, it is important to predict such a transition of ductile to brittle fracture.

To numerically simulate the ductile-brittle transition, Tvergaard and Needleman ${ }^{1)}$ have employed Gurson-Tvergaard-Needleman (GTN) model $^{2)}$ to represent the plastic deformation along with void nucleation, growth and coalescence and also evaluated the maximum principal stress to realize the onset of the brittle fracture. Moreover, Tanguy et $\mathrm{a}^{3)}$ have utilized Rousselier model ${ }^{4)}$ that has capability of the ductile fracture similar with the GTN model and employed Beremin model ${ }^{5)}$ to stochastically predict the brittle fracture. Although these previous studies have successfully predicted the onset of the brittle fracture, it is difficult to simulate the crack propagation along with the transition from ductile to

\footnotetext{
${ }^{*}$ Received: 2019.11.21, Presented at Visual-JW or WSE 2019

** Student Member, Graduate School of Systems and Information Engineering, University of Tsukuba

${ }^{* * *}$ Member, Department of Engineering information and Systems, University of Tsukuba

${ }^{* * * *}$ Member, Joining and Welding Research Institute, Osaka University

***** Member, International Research Institute of Disaster Science, Tohoku University
}

brittle fracture by utilization of these models independent with each other.

On the other hand, a cohesive traction-separation law that represents the stress release process associated with crack opening is often employed to realize the crack propagation. In fact, Pandofi et $\mathrm{al}^{6)}$ have numerically simulated the rapid propagation of the brittle crack and compared with experiments. As a result, the time before the crack nucleates and the path of the propagating crack can be realized by cohesive traction-separation law.

The objective of this study is to propose an improved Gurson model by combining with cohesive traction-separation law based on the conception of the cohesive-traction embedded damage model ${ }^{7)}$ to realize crack propagation associated with transition from ductile to brittle fracture. Throughout several numerical examples using a notched bar under different temperatures, the capability of the proposed model is demonstrated.

\section{An improved Gurson model with cohesive traction-} separation law

\subsection{Formulation of an improved Gurson model with cohesive traction-separation law}

To embed cohesive cracks into material constitutive law, the apparent strain due crack opening displacement normal to $i$-th direction $w^{(i)}$ is defined as

$$
\boldsymbol{\varepsilon}^{\mathrm{W}}=\sum_{i=1}^{N^{\mathrm{dim}}} \frac{w^{(i)}}{h^{(i)}} \boldsymbol{e}^{(i)} \otimes \boldsymbol{e}^{(i)},
$$


where $h^{(i)}$ is characteristic length, $N^{\mathrm{dim}}$ is the spatial dimension and $\boldsymbol{e}^{(i)}$ is principal stress direction. By introduction of the apparent strain, Cauchy stress is given as

$$
\sigma=\mathbb{C}_{\text {iso }}^{\mathrm{e}}: \varepsilon^{\mathrm{e}}=\mathbb{C}_{\text {iso }}^{\mathrm{e}}:\left(\varepsilon-\varepsilon^{\mathrm{p}}-\varepsilon^{\mathrm{w}}\right),
$$

where $\varepsilon$ is total strain, $\varepsilon^{\mathrm{e}}$ is elastic strain, $\varepsilon^{\mathrm{p}}$ is plastic strain and $\mathbb{C}_{\text {iso }}^{\mathrm{e}}$ is the 4-th order isotropic elasticity tensor. Also, to postulate the local balance on the crack surface between the principal stress $\sigma^{(i)}$ and the cohesive traction $t^{(i)}$, the following conditional equation is obtained as

$$
\begin{aligned}
g^{\mathrm{w}(i)} & =\sigma^{(i)}-t^{(i)}\left(w^{(i)}\right) \\
& =\boldsymbol{e}^{(i)} \cdot\left[\mathbb{C}_{\mathrm{iso}}^{\mathrm{e}}:\left(\varepsilon-\varepsilon^{\mathrm{p}}-\varepsilon^{\mathrm{w}}\right)\right] \boldsymbol{e}^{(i)}-t^{(i)}\left(w^{(i)}\right)=0 .
\end{aligned}
$$

Here, the relation between the cohesive traction and crack opening displacement is identified by a specific cohesive tractionseparation law as written in Subsection 2.3.

\subsection{Yield function of Gurson model for ductile fracture}

Gurson model ${ }^{8)}$ is employed to realize effect of voids on the plastic deformation. The yield function of Gurson model is given by void volume fraction $f$ as

$$
g^{\mathrm{p}}=\sigma_{\mathrm{eq}}^{2}-\left(a \sigma_{y}\right)^{2}=0 .
$$

Here, $\sigma_{\mathrm{eq}}$ is equivalent stress and $a$ is defined as

$$
a=\sqrt{1+f^{2}-2 f \cosh \left(\frac{3}{2} \frac{\sigma_{\mathrm{v}}}{\sigma_{\mathrm{y}}}\right)},
$$

where $\sigma_{\mathrm{v}}$ is hydrostatic stress and the yield stress $\sigma_{y}$ with dependence of temperature in Reference ${ }^{9)}$ is given as

$$
\begin{aligned}
\sigma_{\mathrm{y}} & =\sigma_{\mathrm{y} 0}+\kappa \\
& =\sigma_{\mathrm{y} 0}+Q_{1}\left(1-\exp \left(-b_{1} R\right)\right)+Q_{2}\left(1-\exp \left(-b_{2} R\right)\right)
\end{aligned}
$$

Here, $\sigma_{\mathrm{y} 0}$ is initial yield stress, $Q_{1}$ and $b_{1}$ are coefficients depending on temperature, $Q_{2}$ and $b_{2}$ are material constants and $R$ is the accumulated plastic strain. Then, the plastic flow rule and evolution equation of hardening coefficient is obtained from derivation of Eq. (4) by $\sigma$ and $\kappa$ as

$$
\begin{aligned}
& \dot{\boldsymbol{\varepsilon}}^{\mathrm{p}}=\dot{\gamma}^{\mathrm{p}}\left(3 \sigma_{\mathrm{d}}+b \sigma_{\mathrm{y}} \mathbf{I}\right), \\
& \dot{R}=\dot{\gamma}^{\mathrm{p}}\left(3 b \sigma_{\mathrm{v}}+2 a^{2} \sigma_{\mathrm{y}}\right),
\end{aligned}
$$

where $(\bullet)$ is time derivative of $(\bullet), \dot{\gamma}^{\mathrm{p}}$ is plastic multiplier, $\sigma_{\mathrm{d}}$ is the deviatoric stress and $\mathbf{I}$ is identity matrix. Here, $b$ is defined as

$$
b=f \sinh \left(\frac{3}{2} \frac{\sigma_{\mathrm{v}}}{\sigma_{\mathrm{y}}}\right) .
$$

Also, $\sigma_{\mathrm{v}}$ is obtained by the hydrostatic modulus $K$ and additive decomposition of Eq. (7) as

$$
\dot{\sigma}_{\mathrm{v}}=\dot{\gamma}^{\mathrm{p}} b K \sigma_{\mathrm{y}} \text {. }
$$

On the other hand, the evolution equation of the void volume fraction is additively decomposed into the nucleation and growth

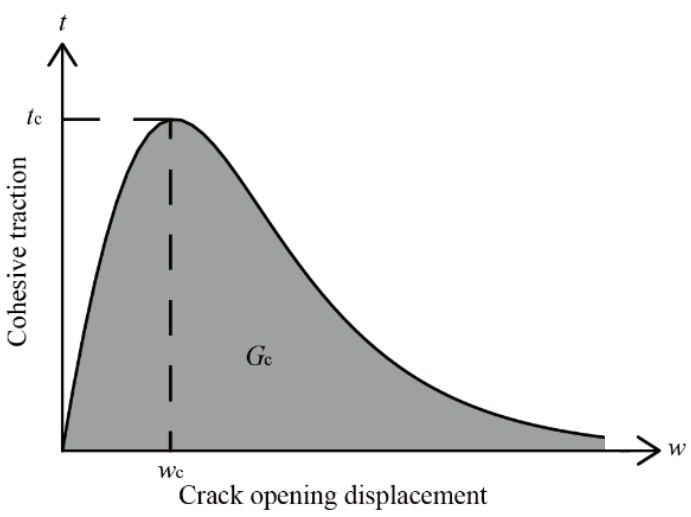

Fig. 1 Relationship between effective cohesive traction and crack opening displacement

term $\dot{f}_{\text {nucleation }}$ and $\dot{f}_{\text {growth }}$ as

$$
\dot{f}=\dot{f}_{\text {nucleation }}+\dot{f}_{\text {growth }} \text {. }
$$

The nucleation term is identified by the accumulated plastic strain as

$$
\dot{f}_{\text {nucleation }}=A \dot{R},
$$

where $A$ is the material constant. On the other hand, the growth term is obtained from the mass conservation law as

$$
\dot{f}_{\text {growth }}=(1-f) \dot{\varepsilon}_{\mathrm{v}}^{\mathrm{p}},
$$

where $\varepsilon_{\mathrm{v}}^{\mathrm{p}}$ is hydrostatic plastic strain.

Therefore, five kinds of the consistency conditions are simultaneously solved for equilibrium conditions Eq. (3), yield function of Gurson model Eq. (4), evolution equation of hardening coefficient Eq. (8), evolution equation of hydrostatic stress Eq. (10) and evolution equation of void volume fraction Eq. (11).

\subsection{Cohesive traction-separation law for brittle fracture}

The cohesive traction-separation law represents stress release process associated with crack opening displacement and cohesive traction between two opposite crack surfaces. In this study, the exponential type proposed by Rice and Wang ${ }^{10)}$ is employed. The cohesive potential of Rice and Wang is defined as

$$
\Psi(w)=G_{\mathrm{c}}\left[1-\left(1+\frac{w}{w_{\mathrm{c}}}\right) \exp \left(-\frac{w}{w_{\mathrm{c}}}\right)\right],
$$

where $G_{\mathrm{c}}$ is critical energy release rate and $w_{\mathrm{c}}$ is critical crack opening displacement. The cohesive traction is obtained from derivation of cohesive potential by the crack opening displacement as

$$
t^{(i)}(w)=\frac{\partial \Psi}{\partial w}=\frac{G_{\mathrm{c}}}{w_{\mathrm{c}}}\left(\frac{w^{(i)}}{w_{\mathrm{c}}}\right) \exp \left(-\frac{w^{(i)}}{w_{\mathrm{c}}}\right) .
$$

Here, the critical energy release rate is area surrounded by curve and horizontal axis in Fig. 1, which is given by integration of Eq. (15) by $w^{(i)}$ as 


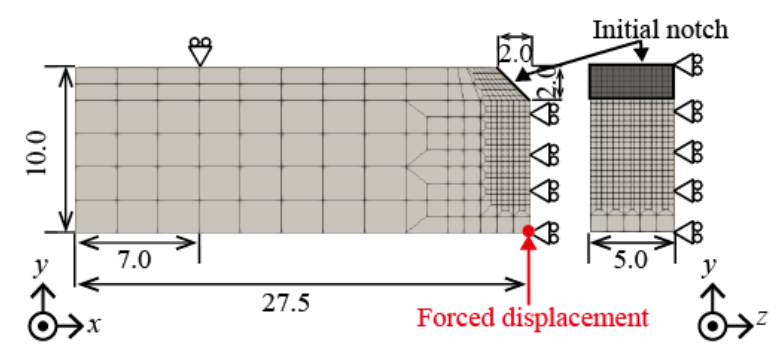

Fig. 2 Numerical model with boundary conditions

Table. 1 Material constants of JIS SFVQ1A (ASTM A508)

\begin{tabular}{|c|c|c|c|}
\hline Elastic modulus[GPa] & 492.3 & $t_{c}[\mathrm{MPa}]$ & 2400.0 \\
\hline Poisson's ratio & 0.3 & $G_{c}\left[\mathrm{~kJ} / \mathrm{m}^{2}\right]$ & 84.8 \\
\hline Initial void volume function & 0.0 & $Q_{2}^{3)}$ & 470.0 \\
\hline$A^{3)}$ & $3.8 \times 10^{-2}$ & $b_{2}^{3)}$ & 1.7 \\
\hline \multicolumn{4}{|c|}{$\begin{aligned} \sigma_{y 0}= & \left(5.0 \times 10^{-8}\right) T^{4}-\left(2.0 \times 10^{-5}\right) T^{3} \\
& +\left(3.1 \times 10^{-3}\right) T^{2}-\left(5.7 \times 10^{-1}\right) T+4.8 \times 10^{2}\end{aligned}$} \\
\hline \multicolumn{4}{|c|}{$Q_{1}=\left(6.0 \times 10^{-4}\right) T^{2}-\left(3.1 \times 10^{-1}\right) T+1.5 \times 10^{2}$} \\
\hline \multicolumn{4}{|c|}{$b_{1}=\left(1.5 \times 10^{-1}\right) T+3.1 \times 10$} \\
\hline
\end{tabular}

$$
G_{\mathrm{c}}=\int_{0}^{\infty} t^{(i)} \mathrm{d} w^{(i)}=\mathrm{e} t_{\mathrm{c}} w_{\mathrm{c}},
$$

where $t_{\mathrm{c}}$ is critical cohesive traction and $\mathrm{e}$ is natural logarithm.

As shown in Fig. 1, after the crack opening displacement reaches its critical value, cohesive traction asymptotically decreases toward to zero along with nucleation and propagation of crack surface.

\section{Numerical example}

To demonstrate capability of the improved Gurson model in this study, the numerical simulation is conducted by comparison with experimental results ${ }^{11)}$ in accordance with French standard for impact testing of Charpy specimens (NF EN 10045-1) under different temperatures and quasi-static conditions that striker speed is $1.0 \mu \mathrm{ms}^{-1}$.

Figure 2 shows the corresponding FE model, which is the one fourth of a notched bar in consideration of the symmetric condition. The forced displacement of $5.5 \mathrm{~mm}$ is applied at red mark in Fig. 2. The material constants of JIS SFVQ1A that is equivalent to ASTM A508 are given in Table 1 and the dependence of the temperature $T$ is determined by approximate function from the experiments ${ }^{11)}$. Also, the material constant $A$ in Eq. (12) is set to 0.038 as Reference $^{3)}$ at specific plastic strain range $(0.25 \leq R \leq 0.85)$, otherwise it keeps 0.0 .

Figure 3 shows the load-displacement curves obtained from the numerical simulations with the experiments ${ }^{11)}$ under different temperatures. From the load-displacement curves until the load

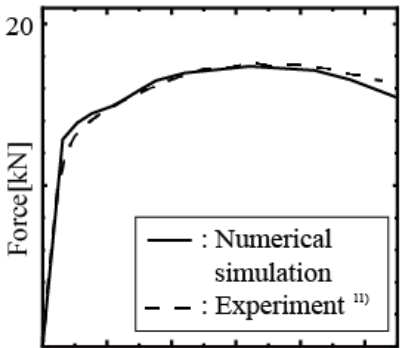

0 Forced displacement[mm] 5.0

(a) $-60{ }^{\circ} \mathrm{C}$

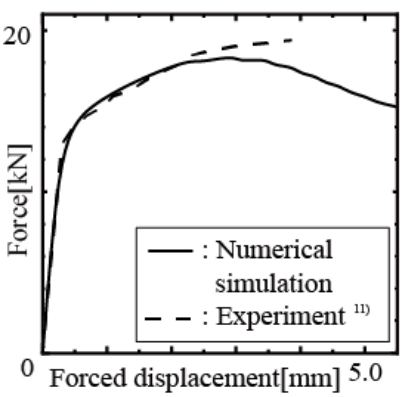

(c) $-100{ }^{\circ} \mathrm{C}$

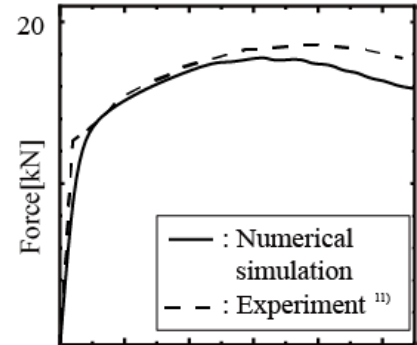

0 Forced displacement[mm] 5.0

(b) $-80^{\circ} \mathrm{C}$

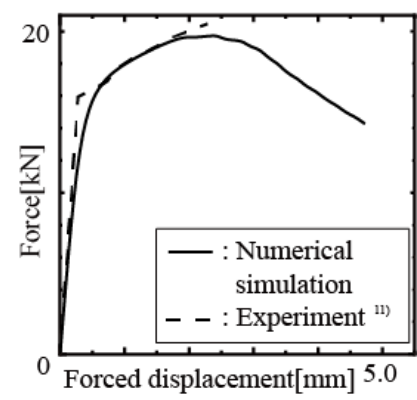

(d) $-140{ }^{\circ} \mathrm{C}$
Fig. 3 Comparison of load-displacement curves at $-60,-80,-100$ and $-140{ }^{\circ} \mathrm{C}$ with experiment ${ }^{11)}$

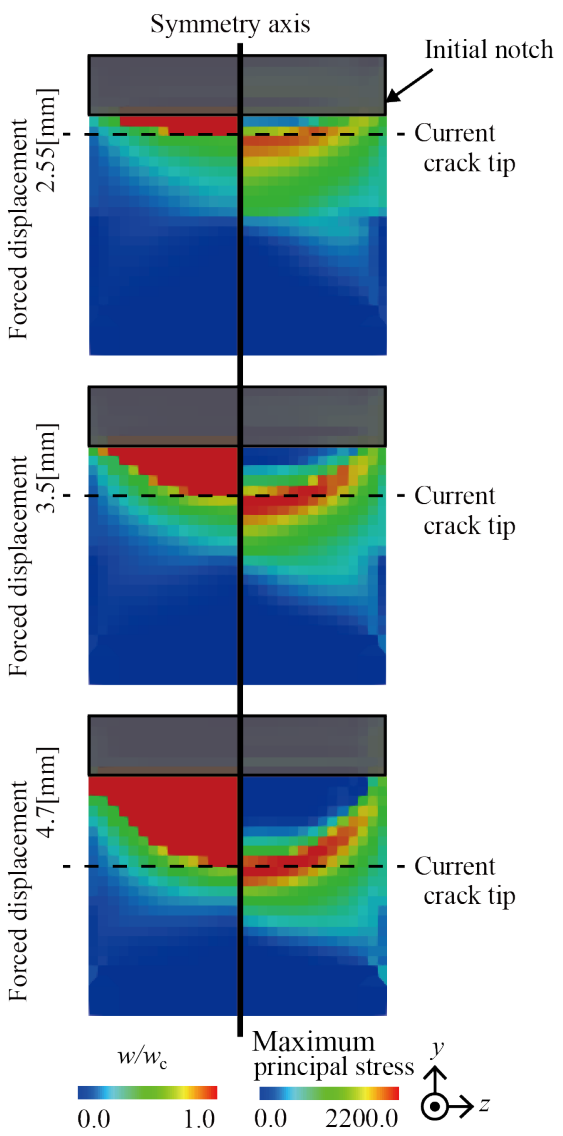

Fig. 4 Distributions of state variables on crack surface at $-140{ }^{\circ} \mathrm{C}$. (Left side shows ratio of crack opening displacement to its critical value and right side is maximum principal stress.) 


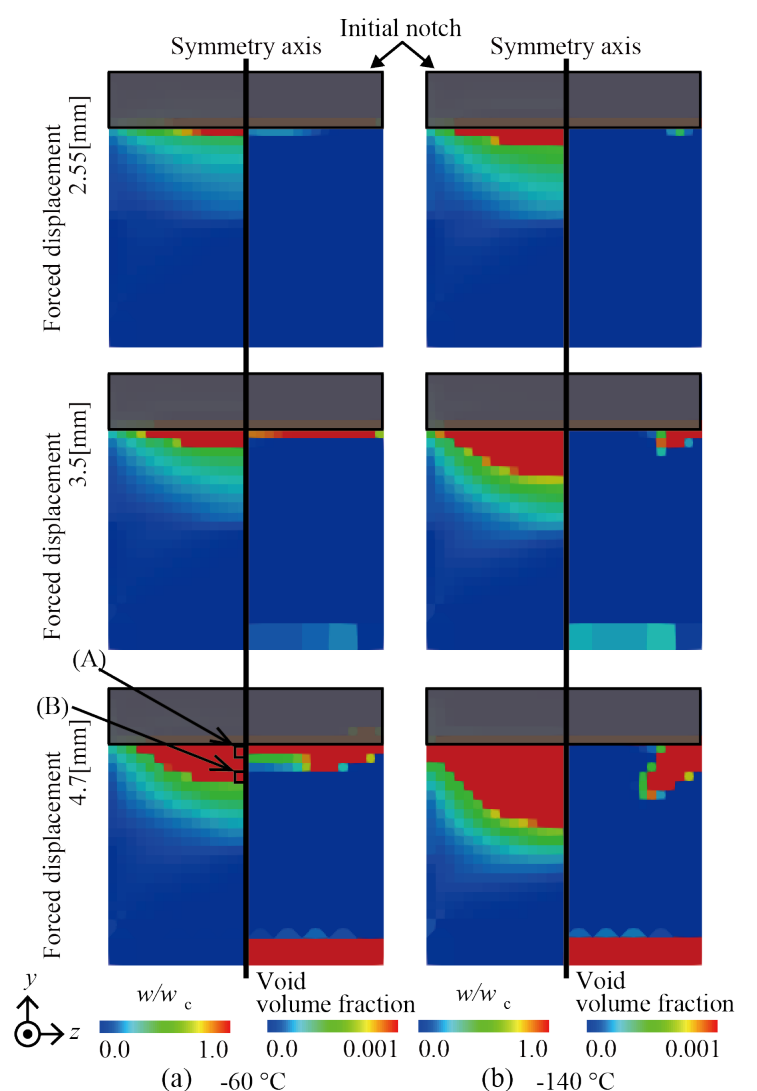

(a) $-60^{\circ} \mathrm{C}$

(b) $-140{ }^{\circ} \mathrm{C}$

Fig. 5 Distributions of state variables on crack surface at $-60^{\circ} \mathrm{C}$ and $-140{ }^{\circ} \mathrm{C}$. (Left side shows ratio of crack opening displacement to its critical value and right side is maximum principal stress.)

attains the tensile strength, the hardening behaviors obtained from the numerical simulation are coincident with the experimental one, respectively, owing to the yield stress depending on temperature given in Eq. (6) and Table 1. Also, the change of the tensile strength and the breaking elongation with the temperature is realized by the numerical simulations, although both the energy release rate and the critical cohesive traction corresponding to the tensile strength are assumed to be constant in the proposed model. In fact, as the temperature decreases, the tensile strength macroscopically increases as shown in the numerical results, while the breaking elongation becomes small. This is because the elastic domain becomes larger in the whole specimen due to the increase of the yield stress as the temperature decreases. It can be seen, however, that the predicted tensile strengths by the numerical simulations underestimate the experimental results at $-100{ }^{\circ} \mathrm{C}$ and $-140{ }^{\circ} \mathrm{C}$. Thus, to more accurately reproduce the force-displacement curve at lower temperature, we need to consider introduction of the dependence on the temperature into the energy release rate or the critical cohesive traction, or both of them.

Figure 4 shows the result at $-140{ }^{\circ} \mathrm{C}$ with the distribution of the ratio of crack opening displacement to its critical value and the

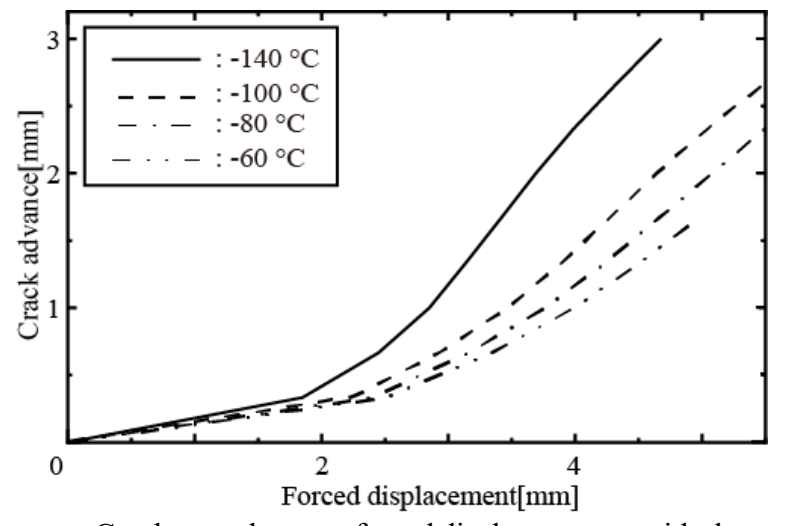

Fig. 6 Crack growth rate to forced displacement at mid-plane at $-60,-80,-100$ and $-140{ }^{\circ} \mathrm{C}$

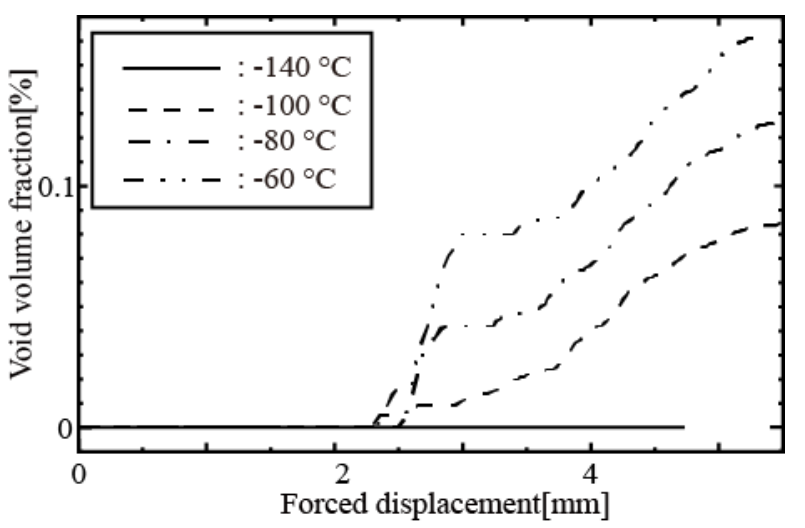

Fig. 7 Development of void volume fraction at initial notch tip at $-60,-80,-100$ and $-140{ }^{\circ} \mathrm{C}$

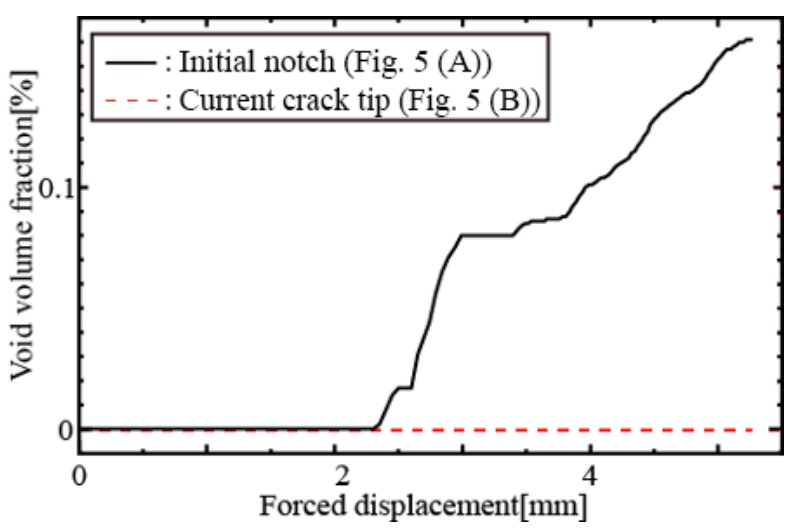

Fig. 8 Development of void volume fraction at initial and current crack tip

maximum principal stress on the crack surface. As can be seen from Fig. 4, the stress concentrates at the current cruck tip, while the stress release occurs at the finite element where the crack opening displacement reaches its critical value crack opening displacement.

To compare between the result at $-60{ }^{\circ} \mathrm{C}$ and $-140{ }^{\circ} \mathrm{C}$, the distribution of the ratio of the crack opening displacement to its critical value and the void volume fraction is shown in Fig. 5 (a) and (b), respectively. From these figures, the crack propagation at $-140{ }^{\circ} \mathrm{C}$ is faster than one at $-60^{\circ} \mathrm{C}$ as shown in the left side of Fig. 5. Here, Fig. 6 shows the crack growth rate to the forced 
displacement at the mid-plane under several temperatures. As can be seen from Fig. 6, the crack growth rate accelerates with the decrease in temperature. It is noted here that the additional acceleration will occur if the dependence of the energy release rate on the temperature is introduced into the proposed model.

From right side of Fig. 5, the void nucleates at the initial notch tip on both mid-plane and free-surface side at $-60{ }^{\circ} \mathrm{C}$, whereas it only occurs at free-surface side at $-140{ }^{\circ} \mathrm{C}$. It is reported in Reference $^{12)}$ that the percentage of the dimple in the fracture surface increases as the temperature rises from $-130{ }^{\circ} \mathrm{C}$ whereas the crack propagation below $-130{ }^{\circ} \mathrm{C}$ is mainly caused by the cleavage fracture. Also, Fig. 7 shows the development of the void volume fraction at the initial notch tip depicted as the element (A) in Fig. 5 under several temperatures. As shown in Fig. 7, the void volume faction increases with the rise in temperature from $-140^{\circ} \mathrm{C}$. Finally, to compare the fracture mechanism between the initial and the current crack tip at $-60{ }^{\circ} \mathrm{C}$, Fig. 8 shows the change of the void volume fraction at the element (A) and (B) depicted in Fig. 5. From this figure, the void volume fraction become large value around initial notch due to ductile fracture whereas it is almost zero at the current crack tip. This result implicates the nucleation and propagation of the crack is initially caused by the ductile fracture and then the subsequent propagation arises due to the transition into the brittle fracture, which has been observed in the experiment ${ }^{9)}$.

\section{Conclusions}

To simulate the ductile-brittle transition, the improved Gurson model has been proposed by combining with the cohesive tractionseparation law. Throughout the numerical examples at different temperatures, it has been confirmed that the proposed model provides us to realize load-displacement curves depending on temperature along with the ductile-brittle fracture as observed in the experiment ${ }^{11)}$. Also, the proposed model has represented changes of crack propagation rate and void volume fraction by depending on temperature. Moreover, the proposed model has enabled us to realize the crack propagation associated with the transition from the ductile to brittle fracture at $-60{ }^{\circ} \mathrm{C}$

\section{Acknowledgements}

This work was supported by JSPS KAKENHI Grant Numbers JP17K17627.

\section{Reference}

1) V. Tvergaard and A. Needleman.: An analysis of the temperature and rate dependence of Charpy $\mathrm{V}$-notch energies for a high nitrogen steel, Int. J. Fract., 37 (1988), 197-215.

2) V. Tvergarrd and A. Needleman: Analysis of the cup-cone fracture in a round tensile bar, Acta Metall. 32 (1984), 157-169.

3) B. Tanguy, J. Besson, R. Piques and A. Pineau: Ductile to brittle transition of an A508 steel characterized by Charpy impact test Part II: modeling of the Charpy transition curve, Eng. Fract. Mech., 37 (2005), 423-434.

4) G. Rousselier: Ductile fracture models and their potential in local approach of fracture, Nucl. Eng. Des., 105 (1987), 97-111.

5) F.M. Beremin: A local criterion for cleavage fracture of a nuclear pressure vessel steel, Metall. Trans. A, 14 (1083), 2277-2287.

6) A. Pandolfi, P.R. Guduru, M. Ortiz, A.J. Rosakis: Three dimensional cohesive-element analysis and experiments of dynamic fracture in C300 steel, Int. J. Sol. Struct., 37 (1999), 3733-3760.

7) Y. Shintaku and K. Terada: Cohesive-force embedded damage model and its application to crack propagation analysis (in Japanese), Transactions of JSCES, 2016 (2016), 20160011.

8) A.L. Gurson: Continuum theory of ductile rupture by void nucleation and growth: Part I - yield criteria and flow rules for porous ductile media, J. Eng. Mater. Technol., 99 (1977), 2-15.

9) B. Tanguy, R. Piques, L. Laiarinandrasana and A. Pineau: Mechanical behavior of A508 steel based on double nonlinear viscoplastic constitutive equation, EUROMAT 2000, (2000), 499504.

10) J.R. Rice and J. Wang: Embrittlement of interfaces by solute segregation, Mater. Sci. Eng., A102 (1989), 23-40.

11) B. Tanguy, J. Besson, R. Piques and A. Pineau: Ductile to brittle transition of an A508 steel characterized by Charpy impact test Part I : experimental results, Eng. Fract. Mech., 72 (2005), 49-72.

12) J. Watanabe, T. Iwadate, Y. Tanaka, T. Yokobori and K. Ando: Fracture toughness in the transition region, Eng. Fract. Mech., 28(1987),589-600. 